\title{
Contributors to the 2019 IMIA Yearbook of Medical Informatics
}

\author{
Editors: \\ Kate Fultz Hollis \\ Oregon Health \& Science University \\ Portland, OR, USA \\ fultzhol@ohsu.edu

\begin{tabular}{|c|}
\hline $\begin{array}{l}\text { Brigitte Séroussi } \\
\text { Sorbonne University \& LLMICS UMR_S } 1142 \\
\text { Tenon Hospital, AP-HP } \\
\text { Paris, France } \\
\text { brigitte.seroussi@aphp.fr }\end{array}$ \\
\hline $\begin{array}{l}\text { Lina F. Soualmia } \\
\text { Normandie University, UNIROUEN, LITIS } \\
\text { Roven, France } \\
\text { Lina.Soualmia@chu-roven.fr }\end{array}$ \\
\hline
\end{tabular} \\ Editorial Assistant: \\ Martina Hutter \\ Heidelberg University Hospital - ZIM \\ Heidelberg, Germany \\ yearbook@imia-services.org
}

Best Papers Editor:

Adrien Ugon

ESIEE-Paris

adrien.ugon@esiee.fr

Advisory Board:

Reinhold Haux

Peter L. Reichertz Insitituef for Medical Informatics

of TU Braunschweig and Hannover

Medical School

Brounschweig, Germany

reinhold.haux@plri.de

Vittor Maojo

Universidad Politécnica de Madrid

Departamento de Inteligencia Arificicial

Facultad de Informatica

Madrid, Spain

vmaojo@fi.upm.es

George Mihalas

University of Medicine and Pharmacy

Dept. of Medical Informatics

Timisoara, Romania

mihalas@EFMl.info

\begin{tabular}{|c|c|}
\hline $\begin{array}{l}\text { Section Editors: } \\
\text { Christian Baumgartner } \\
\text { Graz University of Technology } \\
\text { Graz, Austria } \\
\text { christian.boumgartner@tugraz.ot }\end{array}$ & $\begin{array}{l}\text { Werner Hackl } \\
\text { UMIT - Private University for Health Sciences, } \\
\text { Medical Informatics and Technology } \\
\text { Hall in Tirol, Austria } \\
\text { werner.hack@ @umit.ot }\end{array}$ \\
\hline $\begin{array}{l}\text { Eta S. Berner } \\
\text { Department of Health Services Administration } \\
\text { University of Alabama ot Birmingham } \\
\text { Birmingham, AL, USA } \\
\text { eberner@uab.edu }\end{array}$ & $\begin{array}{l}\text { Alexander Hoerbst } \\
\text { UMIT - Private University of Health Sciences, } \\
\text { Medical Informatics and Technology } \\
\text { Hall in Tirol, Austria } \\
\text { alexander.hoerbst@umit.at }\end{array}$ \\
\hline $\begin{array}{l}\text { Meryl Bloomrosen } \\
\text { Premier healthcare alliance } \\
\text { Washington, DC } \\
\text { meryl_bloomrosen@premierinc.com }\end{array}$ & $\begin{array}{l}\text { William Hsu } \\
\text { University of California Los Angeles } \\
\text { Los Angeles, CA, USA } \\
\text { whsu@mednet.ucla.edu }\end{array}$ \\
\hline $\begin{array}{l}\text { Jacques Bouaud } \\
\text { DRCI/AP-HP \& LIMICS UMR_S } 1142 \\
\text { Paris, France } \\
\text { jacques.bouaud@aphp.fr }\end{array}$ & $\begin{array}{l}\text { Jianying Hu } \\
\text { IBM Research } \\
\text { Yorktown Heights, New York, USA } \\
\text { jyhu@us.ibm.com }\end{array}$ \\
\hline $\begin{array}{l}\text { Jean Charlet } \\
\text { DRCI/AP-HP \& LIMICS UMR_S } 1142 \\
\text { Paris, France } \\
\text { jean.charlet@upmc.fr }\end{array}$ & $\begin{array}{l}\text { Gretchen Purcell Jackson } \\
\text { IBM Wotson Health } \\
\text { Vanderbilt University Medical Center } \\
\text { Nashiille, TN, USA } \\
\text { gretchen.jackson@ibm.com }\end{array}$ \\
\hline $\begin{array}{l}\text { Sebastien Cossin } \\
\text { Bordeaux University \& Inserm UMR } 1219 \\
\text { Bordeauxx,France } \\
\text { sebastien.cossin@u-bordeaux.fr }\end{array}$ & $\begin{array}{l}\text { greerchen.jockson@orom.com } \\
\text { Dipak Kalra } \\
\text { The European Institute for Innovation } \\
\text { through Health Data (i HD) }\end{array}$ \\
\hline \multirow{2}{*}{$\begin{array}{l}\text { Christel Daniel } \\
\text { WIND-DSI/AP-HP \& LIMICS UMR_S } 1142 \\
\text { Paris, France } \\
\text { christel.daniel@aphp.fr }\end{array}$} & $\begin{array}{l}\text { Gent, Belgium } \\
\text { dipak.kalra@i-hd.eu }\end{array}$ \\
\hline & $\begin{array}{l}\text { Vassilis Koutkias } \\
\text { Institute of Applied Biosciences }\end{array}$ \\
\hline \multirow{2}{*}{$\begin{array}{l}\text { Thomas M. Deserno } \\
\text { Peter L. Reichertz Institute for Medical Informatics } \\
\text { of TU Braunschweig and Hannover } \\
\text { Medical School } \\
\text { Braunschweig, Germany } \\
\text { thomas.deserno@plri.de }\end{array}$} & $\begin{array}{l}\text { Thermi, Thessaloniki, Greece } \\
\text { vkoutkias@@certh.gr }\end{array}$ \\
\hline & $\begin{array}{l}\text { Annie Y. S. Lau } \\
\text { Centre for Health Informatics } \\
\text { Australian Institute of Health Innovation }\end{array}$ \\
\hline \multirow{2}{*}{$\begin{array}{l}\text { Ferdinand Dhombres } \\
\text { Sorbonne University \& LMIICS UMR_S } 1142 \\
\text { Trousseau Hospital, AP-HP } \\
\text { Paris, France } \\
\text { ferdinand.dhombres@aphp.fr }\end{array}$} & $\begin{array}{l}\text { Macquarie University, Australia } \\
\text { annie.lau@mq.edu.au }\end{array}$ \\
\hline & $\begin{array}{l}\text { Debra Patt } \\
\text { Texas Oncology } \\
\text { Dell Medical School }\end{array}$ \\
\hline \multirow{2}{*}{$\begin{array}{l}\text { Natalia Grabar } \\
\text { CNRS, Univ. Lille, UMR } 8163 \\
\text { STL-Savoirs Textes Langage } \\
\text { Lille, France } \\
\text { natalia.grabar@univ-lille.fr }\end{array}$} & $\begin{array}{l}\text { Austin, Texas } \\
\text { debra.patt@usoncology.com }\end{array}$ \\
\hline & $\begin{array}{l}\text { Sylvia Pelayo } \\
\text { Lille University \& chu Lille }\end{array}$ \\
\hline \multirow{2}{*}{$\begin{array}{l}\text { Cyril Grouin } \\
\text { LlMSI, CNRS, Université Paris Saday } \\
\text { Orsay, France } \\
\text { cyril.grouin@limsi.fr }\end{array}$} & sylvia.pelayo@univ-lille.fr \\
\hline & $\begin{array}{l}\text { Bastien Rance } \\
\text { HEGP/AP-HP \& Universití Paris Descartes } \\
\text { INSERM UMRS } 1138 \\
\text { Paris, France } \\
\text { bastien.rance@aphp.fr }\end{array}$ \\
\hline
\end{tabular}

\author{
Yalini Senathirajah \\ University of Pittsburgh School of Medicine \\ Pittsburgh, USA \\ yalini@pitt.edu \\ Malika Smaï-Tabonne \\ Loria UMR 7503 \\ Université de Lorraine \\ Nancy, France \\ malika.smail@loria.fr \\ Pascal Staccini \\ IRIS Department \\ Université Côte d'Azur \\ Nice, France \\ pascal.staccini@univ-cotedazur.fr
}

Rodolphe Thiébaut

Inserm U1219 \& Inria SISTM

Bordeaux University

Bordeaux, France

rodolphe.thiebaut@u-bordeaux.fr

Jeremy L. Warner

Vanderbilt University

Nashville, TN, USA

jeremy.warner@vumc.org

Regional Editors:

Vajira Dissanayake

APAMI

president@apami.org

Ghislain B. Kovematchoua Tchuitcheu

HELINA

kouematchoua@helina-online.org

Riyad Al Shammari

MENAHIA

riyadalshammari@gmail.com

Karen Greenwood

AMIA

Bethesda, MD, USA

karen@amia.org

Andre Kushniruk

Digital Health Canada: Canada's Health

Informatics Association

Toronto, ON, Canada

andrek@uvic.ca

Christian Lovis

EFMI

christian.lovis@hcuge.ch

IMIA Executive Director:

Elaine Huesing

imia@imia-services.org 